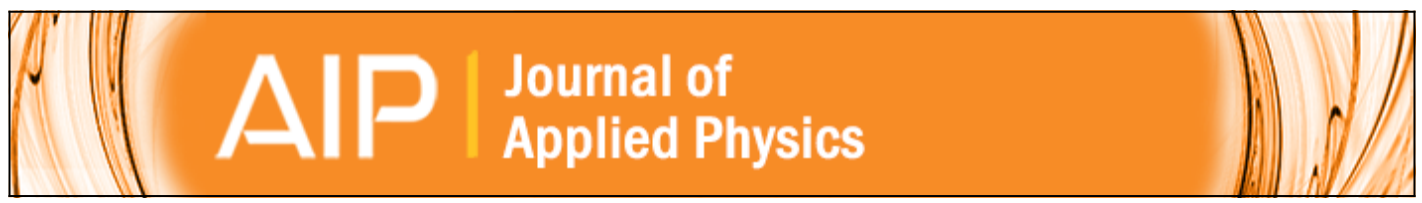

\title{
A Point Focusing XRay Monochromator for the Study of Low Angle Diffraction
}

Leon Shenfil, Warren E. Danielson, and Jesse W. M. DuMond

Citation: Journal of Applied Physics 23, 854 (1952); doi: 10.1063/1.1702319

View online: http://dx.doi.org/10.1063/1.1702319

View Table of Contents: http://scitation.aip.org/content/aip/journal/jap/23/8?ver=pdfcov

Published by the AIP Publishing

\section{Articles you may be interested in}

A pointfocusing small angle xray scattering camera using a doubly curved monochromator of a $\mathrm{W} / \mathrm{Si}$ multilayer

Rev. Sci. Instrum. 67, 688 (1996); 10.1063/1.1146841

PointFocusing TwoCrystal XRay Monochromator for XRay Diffraction

Rev. Sci. Instrum. 28, 1042 (1957); 10.1063/1.1715798

Single Quartz Crystal Point Focusing XRay Monochromator

Rev. Sci. Instrum. 26, 1048 (1955); 10.1063/1.1715183

Latex Particle Size Determination Using Diffraction Peaks Obtained with the Point Focusing XRay Monochromator

J. Appl. Phys. 23, 860 (1952); 10.1063/1.1702320

PointFocus XRay Monochromators for Low Angle Diffraction

Rev. Sci. Instrum. 21, 188 (1950); 10.1063/1.1745527

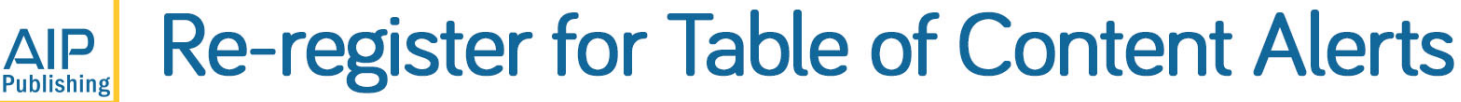

\section{Create a profile.}




\title{
A Point Focusing X-Ray Monochromator for the Study of Low Angle Diffraction*
}

\author{
Leon Shenfil, $\dagger$ Warren E. Danielson, and Jesse W. M. DuMond \\ California Institute of Technology, Pasadena, California.
}

(Received February 18, 1952)

\begin{abstract}
A point focusing $\mathbf{x}$-ray monochromator was designed and constructed for low angle scattering studies. The anastigmatic point focus is achieved by means of two cylindrically bent quartz crystals whose focal circles are mutually perpendicular. The beam, emanating from the copper target of an $x$-ray tube, is reflected in succession, first from the crystal defining the horizontal focal circle, and second from the crystal defining the vertical focal circle following which it comes to a monochromatic point focus of wavelength $1.537 \mathrm{~A}$ $\left(\mathrm{CuK} \alpha_{1}\right)$. The sample to be studied is placed between the second crystal and the point focus, and the scattered beam is detected by means of a photographic film placed at the point focus, at right angles to the undeviated beam, the latter being suppressed by means of an absorber, or allowed to pass through a hole in the film.

Mathematical analysis, in which a ray was traced through the two-crystal system, revealed correctly the shape and size of the point focus, and the possibility of reducing the latter in size by stopping down the beam emerging from the target.
\end{abstract}

\section{INTRODUCTION}

$\mathrm{U}^{\mathrm{P}}$ to the present, most low angle $x$-ray scattering work has been done with x-ray beams monochromatized, if at all, only by means of filters and collimated by means of pinhole or slit systems. An exception to this is the work of Guinier in France, ${ }^{1}$ who with collaborators has used curved crystals to monochromatize and refocus an $x$-ray beam to a line focus. Guinier, following a suggestion by one of the present authors, has even tried two successive crystal reflections, with their focal circles in the same plane, in an effort to reduce the fogging due to incoherent crystal scattering. To our knowledge, however, one of us was the first to propose ${ }^{2}$ a method of effecting two successive curved crystal reflections by means of elastically bent crystal laminas whose focal circles lie in mutually perpendicular planes, the geometry being such that the monochromatic radiation passed by the laminas converges to an anastigmatic point focus. The instrument here described was designed to exploit the possibilities of this proposal.

A list of references to recent work in the field of low angle $\mathrm{x}$-ray diffraction is appended herewith. ${ }^{3-6}$

An endeavor has been made to design the present instrument so as to give high resolution in the low angle scattering range (down to 0.002 radians scattering angle) with a higher total intensity than has been achieved by the use of pinhole systems with comparable

* This work was performed and financed under the joint sponsorship of the ONR and the AEC by contract with the California Institute of Technology.

$\dagger$ Now with the Aerojet Engineering Corporation, Azusa, California.

1 A. Guinier, Ann. phys. 12, 161 (1939); J. chim. phys. 40, 133 (1943); Bull. soc. chim. Belges 57, 286 (1948); A. Guinier and G. Fournet, Compt. rend. 226, 656 (1948).

${ }_{2}^{2}$ J. W. M. DuMond, Rev. Sci. Instr. 21, 188 (1950).

3 R. S. Bear and O. E. A. Bolduan, J. Appl. Phys. 22, 191 (1951);

O. E. A. Bolduan and R. S. Bear, J. Appl. Phys. 20, 983 (1949).

${ }_{4}$ K. L. Yudowitch, J. Appl. Phys. 22, 214 (1951); 20, 1232 (1949); 20, 174 (1949).

C. G. Shull and R. C. Roess, J. Appl. Phys. 18, 295 (1947);

R. C. Roess and C. G. Shull, J. Appl. Phys. 18, 308 (1947).

${ }^{6}$ L. H. Lund and G H. Vineyard, J. Appl. Phys. 20, 593 (1949). resolution. To accomplish this, use has been made of the focusing properties of two thin quartz (crystal) laminas, each of which is bent elastically to a cylindrical shape. These two laminas, which by two successive reflections focus the beam to a monochromatic point, constitute the essential feature of the instrument.

The advantages of a point-focusing monochromator are twofold:

(1) The focal spot plays a similar role in delineating the $x$-ray diffraction pattern to that of the cathoderay spot in producing a picture on a television screen. Thus, when the focal spot is small in comparison to the interesting detail of the pattern, the interpretation of this pattern and the reduction and correction of the data are greatly facilitated. $\neq$

(2) The incoherent radiation scattered by the first crystal (which is illuminated by the entire continuous $\mathrm{x}$-ray spectrum) is much more effectively reduced by the second crystal than is the Bragg-reflected line intensity. Hence the focal plane is relatively freer from unwanted diffuse radiation than in single-crystal or slit systems.

The positions of the two laminas rela tive to the source of $x$-rays are shown in horizontal plan and vertical elevation in Fig. 1. (A perspective line drawing is also shown in Fig. 3 below.) In the plan view of Fig. 1 one can observe the $x$-rays emanating from the point source $S$ towards the first crystal (crystal $A$ ), the position of the latter being such as to reflect the $K \alpha_{1}$ line of $\mathrm{Cu}$. In the absence of the second crystal (crystal $B$ ) the beam comes to a vertical line focus $F F$. The three points $S, A$, and $F_{0}$ define the horizontal focal circle with center at $C$. (The points $A$ and $F_{0}$ refer to the centers of crystal $A$ and of the line focus $F F$, respectively.) It can be shown that the reflected beam appears to come from the virtual line source $F^{\prime} F^{\prime}$

$\ddagger$ The size of the point can be decreased at will by stopping down the beam emerging from the target. Without such stops and with our present $x$-ray tube (Machlett) the point appears slightly elongated with dimensions $0.196 \mathrm{~mm}$ by $1.40 \mathrm{~mm}$. (Distance of scattering sample to focal plane, $66 \mathrm{~cm}$.) 


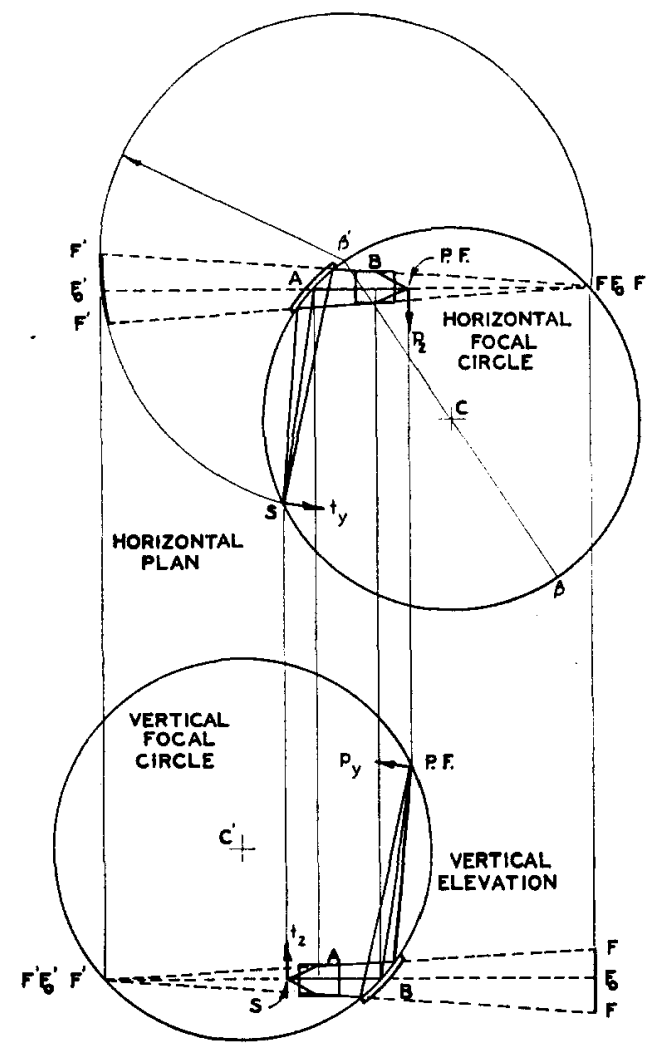

Fic. 1. Two bent crystals arranged to form a point focusing monochromator.

lying in the horizontal plane and having a radius of curvature $\beta^{\prime} S$ as shown. ( $\beta$ is the common center of curvature of the bent atomic planes in crystal $A$, and $\beta^{\prime}$ is diametrically opposite $\beta$ on the focal circle.) Thus, when the second crystal is in position, as is shown in the vertical elevation, the once reflected beam is reflected again to the point focus $P . F$. The three points $F_{0}{ }^{\prime}, B$, and $P . F$. lie on a vertical focal circle with center at $C^{\prime}$ (where again the points $F_{0}^{\prime}$ and $B$ refer to the centers of the virtual line source $F^{\prime} F^{\prime}$ and crystal $B$, respectively). If the latter were superimposed on the horizontal focal circle, then the above three points would coincide, respectively, with $F_{0}, A$, and $S$, the two focal circles being identical in size.

The sample is placed in the twice reflected beam near crystal $B$, and the photographic plate is placed at the point focus, at right angles to the central ray. The low angle diffraction pattern appears on the photographic film as a diffuse distribution of film grain in a small region about the direct beam, the latter being suppressed by means of an absorber, or allowed to pass through a hole in the film.

\section{GEOMETRY OF A SINGLE BENT CRYSTAL}

It is well known ${ }^{7,8}$ that $\mathrm{x}$-rays can be made to focus monochromatically to a line focus by a crystal lamina

${ }^{7}$ J. W. M. DuMond and H. A. Kirkpatrick, Rev. Sci. Instr. 1, $90(1930)$.

${ }^{8}$ H. H. Johansson, Z. Physik 82, 507 (1933). which has been hollowed out, cylindrically, to a given radius and then bent elastically to half that radius. Figure 2 shows a plan view of such a bent crystal with $C$ the center of the focal circle, $S$ a point source of $x$-rays, $F$ the line focus, and $\beta$ the vertical line (or generator) about which the crystal "planes" (cylinders after bending) are concentric. Lines $\overline{S P}$ and $\overline{P F}$ represent the incident and reflected rays (not necessarily in the plane of the focal circle). It is clear that if the arcs $S \beta$ and $\beta F$ are equal, the angles of incidence and reflection $(\pi / 2-\theta)$ are equal and constant for all positions of the point $P$, provided the vertical divergence angle (relative to the plane of the focal circle) is not excessive. The wavelength of the focused beam is given by the Bragg law $\lambda=2 d \sin \theta$, where $d$ is the grating spacing of the atomic reflecting planes. The position of point $S$, relative to the crystal, is such that the $\mathrm{K} \alpha_{1}$ line of copper is reflected in the monochromator here described.

In Fig. 2, the point on the focal circle diametrically opposite $\beta$ is denoted by $\beta^{\prime}$, and the center of the short piece of crystal lamina, $Z Z^{\prime}$, used in the present instrument is denoted by $A$. It should be clear from this figure that a much larger crystal lamina (such as that indicated, partly with lighter lines, between the points $Z Z$ in the figure), with center at $\beta^{\prime}$ could be used to form the monochromatic line focus through $F$. The reason for using only a portion $Z Z^{\prime}$ entirely on one side of $\beta^{\prime}$ will be evident as soon as the two-crystal arrangement is discussed. The angle $2 \alpha$ is a measure of the angular displacement of point $A$ from $\beta^{\prime}$. $\alpha$ is also the angle between the atomic reflecting planes and the crystal face at $A$.
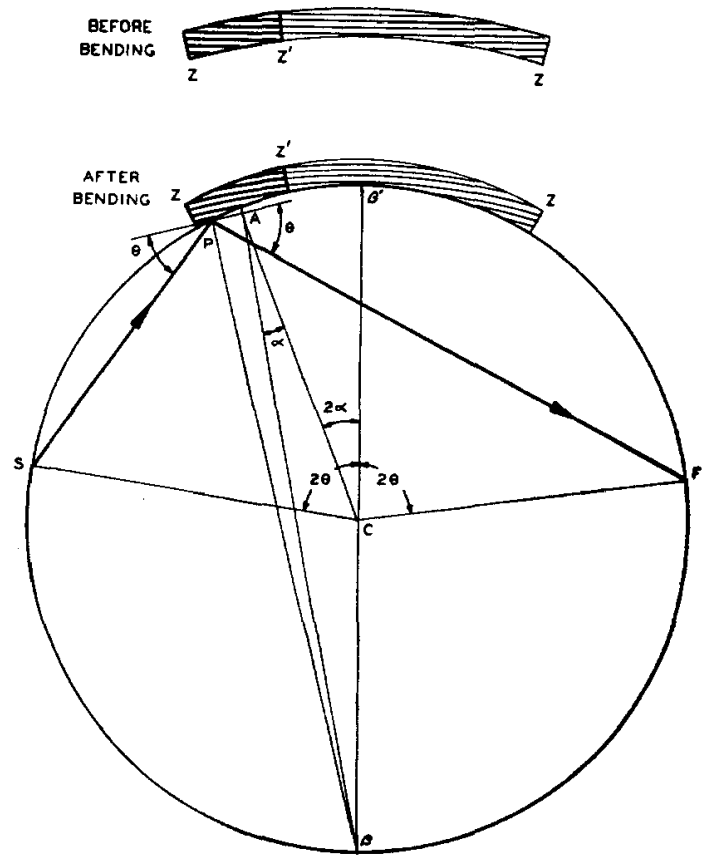

FIG. 2. Geometry of a single bent crystal, cylindrically ground before bending. 


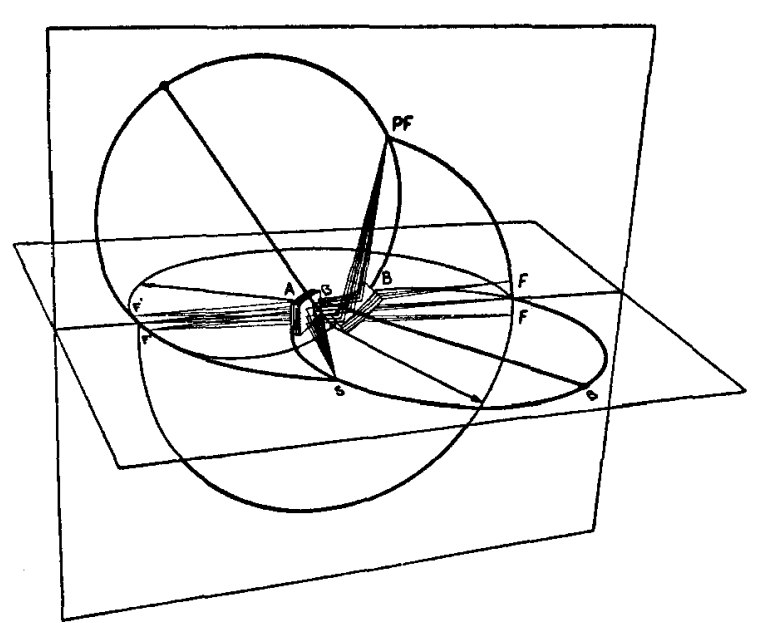

Fig. 3. Perspective line drawing to illustrate the 3-dimensional relationships involved in the geometry of the point focusing $\mathrm{x}$-ray monochromator.

\section{GEOMETRY OF THE POINT-FOCUSING MONOCHROMATOR}

Now let us take a second identical crystal, as is shown in Fig. 1, with the plane of its focal circle perpendicular to the corresponding plane of the first crystal and place it in such a way that the line of intersection of the two planes is $F_{0} F_{0}{ }^{\prime}$. If we let $B F_{0}{ }^{\prime}=A F_{0}$ and if the point $F_{0}{ }^{\prime}$ lies on the focal circle of the second crystal, then the rays striking this crystal will be reflected at the Bragg angle and will come to a point focus.

It should now be evident (1) why only a part, $Z Z^{\prime}$, of the total conceivable crystal $Z Z$ shown in Fig. 2 is retained and (2) why the center $A$ of the crystal $Z Z^{\prime}$ is displaced angularly by an amount $2 \alpha$ from the point $\beta^{\prime}$. Both of these requirements originate from the fact that the first crystal must be kept entirely on one side of the point $\beta^{\prime}$ and the second crystal entirely on the other side so as to avoid mechanical interference between the two crystals or their crystal holders when the two focal circles are correctly mounted to avoid astigmatism. Clearly, the larger the angle $\alpha$ is made, the farther apart the two crystal clamping blocks may be placed. The distance between the centers $A$ and $B$ of the two crystals is given by $4 r \sin \alpha \cos \theta$ where $r$ is the radius of either focal circle.

Figure 3 is a perspective line drawing showing the two mutually perpendicular planes on which the two focal circles are described, together with the two curved crystals and the pencils of $x$-rays diverging from the source and reconverging to the anastigmatic point focus after the two successive reflections. Between the two crystals the beam is astigmatic, appearing to come from one line focus on the circular arc $F^{\prime} F^{\prime}$ and directed so that, were it not for the reflection at the second crystal, the beam would converge in the second line focus on the circular arc $F F$.

\section{SIZE AND SHAPE OF THE POINT FOCUS}

Since in practice one deals with a target of finite size and a spectral distribution ( $\mathrm{K} \alpha_{1}$ line) of finite width, it is reasonable to expect that the point focus will be of a finite size. To calculate this size, it is necessary to trace a general ray through the system. As a result of this analysis we shall find that only certain regions of the focal spot of the $\mathrm{x}$-ray tube are so situated that the monochromator crystals can accept radiation from them within the limitations set by (1) the Bragg law at both crystals, and (2) the natural spectral breadth of the x-ray line. These regions, projected on a plane normal to the mid-ray of the beam from the source to the first crystal, we shall call "the usable projected target area." The size and shape of this area is imaged one-to-one in the focal spot formed after the two-crystal reflections.

In Fig. 4, $S_{0}$ represents the center of the target and $S$ the point at which an arbitrary ray intersects the focal cylinder. $Q$ is the point in which this arbitrary ray intersects the plane through $S_{0}$ normal to the midray, $\overline{S_{0} A}$. The horizontal and vertical displacements of $Q$ from $S_{0}$ are $t_{y}$ and $t_{z}$, respectively. $\S P$ is an arbitrary point on the crystal lamina, not necessarily in the plane of the focal circle. $F_{0}$ is the center of the line focus, and $F$ is the point on the focal cylinder where the general ray $S P F$ intersects it. $2 t$ is the angle $S C S_{0}$ and $-2 \eta$ is the central angle $A C P$, both angles measured in the plane of the focal circle. (Since the focal circle is taken as having unit radius, $2 t=S S_{0}$ so that $t_{y}$ may also be thought of as the projection of the arc $2 t$ on the $t_{y} t_{z}$ plane.) The angle $\theta_{0}$ satisfies the Bragg law $\lambda_{0}=2 d$ $\sin \theta_{0}$ where $\lambda_{0}$ is the center of the $\operatorname{CuK} \alpha_{1}$ line. This angle is to be distinguished from $\theta_{B}$, which is the Bragg angle for a wavelength $\lambda$ falling at some arbitrary point within the natural breadth of the $\mathrm{x}$-ray line whose peak or center is at $\lambda_{0} . \theta$ and $\theta^{\prime}$ are the grazing angles of the general ray at the first and second crystals, respectively. Thus, if the (cylindrical) coordinates of the arbitrary point $P$ are $1,2 \eta, z$, where $z$ is the coordinate normal to the plane of the focal circle and the radius of the focal circle is reduced to unity for convenience, it is not hard to show that the relation between the grazing angle $\theta$ (angle between the general ray and crystal planes at $P$ ) and $\theta_{B}$ is given by

$$
\begin{array}{r}
\theta-\theta_{B}=t-\left[z^{2} \tan \theta_{0} / 8 \sin ^{2}\left(\theta_{0}-\alpha\right)\right] \\
-\left[\left(\lambda-\lambda_{0}\right) / \lambda_{0}\right] \tan \theta_{0},
\end{array}
$$

where the quantities on the right are small compared to $\theta$ or $\theta_{B}$. Furthermore, the grazing angle of the general ray at the second crystal is given by

$$
\theta^{\prime}-\theta_{B}=t^{\prime}-\left[\eta^{2} / 2\right] \tan \theta_{0}-\left[\left(\lambda-\lambda_{0}\right) / \lambda_{0}\right] \tan \theta_{0},
$$

$\$$ The process of "reflection" in the first crystal keeps source and virtual image in the same horizontal plane, consequently the length of $t_{z}$ is equal to that of its image. Hence, $t_{z}$ also represents the vertical displacement of the image of $Q$ from the mid-ray incident on the second crystal just as $t_{y}$ is the horizontal displacement of $Q$ from the mid-ray incident on the first crystal. 
where $2 t^{\prime}=t_{z} / \sin \left(\theta_{0}+\alpha\right)$ is the angle in the vertical focal circle measured about $C^{\prime}$ as center from $F_{0}^{\prime}$ to the point of intersection of the general once-reflected ray with this circle (just as $2 t$ in the horizontal focal circle is the angle about $C$ from $S_{0}$ to $S$ ). The above two equations were derived for the case of cylindrically ground crystals prior to bending, henceforth to be called the exact focusing case. This is to be distinguished from the approximate or inexact focusing case in which one starts with flat crystals prior to bending.

In order that the general ray be reflected simultaneously from both crystals in succession, the following equation must be satisfied:

$$
\theta-\theta_{B}=\theta^{\prime}-\theta_{B}=0 \text {, }
$$

where the equality holds to within the diffraction pattern width ("dynamical" theory of Darwin or Ewald). This width in the case of $\mathrm{CuK} \alpha_{1}$ radiation and the (310) planes of quartz is 2 seconds of arc.

In Eq. (2) a number of approximations were made, namely the omission of terms of order higher than $z^{2}, \eta^{2}$, or $\eta z$, where $\eta$ and $z$, because of the limited size of the crystals, do not exceed $1 / 80$ and $1 / 60$ radians, respectively. The omitted terms are of the order of magnitude of the diffraction pattern width which is taken as the limit of negligibility.

Subtracting Eq. (2) from Eq. (1), we get the dependence of $\eta$ and $z$ on $t$ and $t^{\prime}$ alone,

$$
\left(\eta^{2} / 2\right) \tan \theta_{0}-\left(z^{2} \tan \theta_{0}\right) /\left(8 \sin ^{2}\left(\theta_{0}-\alpha\right)\right)=l^{\prime}-t .
$$

If the target of the x-ray tube is so small that $t$ and $t^{\prime}$ can be neglected or if $t^{\prime}-t=0$ in the above equation, the area of the crystal which is contributing to the point focus degenerates into two lines whose widths ( $3 \mathrm{~mm}$ ) correspond to the diffraction pattern width and whose equations are

$$
z= \pm 2 \eta \sin \left(\theta_{0}-\alpha\right)
$$

along which the wavelength decreases with increasing $|z|$, as is given by Eq. (1).

Since $2 \eta$ and $z$ can be considered as the two-dimensional coordinates of the point $P$ relative to the center (A) of the first crystal, it is not hard to see that Eq. (4) represents two families of hyperbolas and Eqs. (5) represent their two asymptotes, with $t^{\prime}-t$ playing the role of a parameter.

The extreme values of $t^{\prime}-t$ occur when $\eta=\eta_{\max }$ $=1 / 80 \mathrm{rad}, z=0$ and when $z=z_{\max }=1 / 60 \mathrm{rad}, \eta=0$. When substituted in Eq. (4) together with $\theta_{0}=40.7^{\circ}$ and $\alpha=2.1^{\circ}$, the range of $t^{\prime}-t$ becomes

$$
-0.04 \leqslant t^{\prime}-t \leqslant 0.04 \mathrm{~mm}
$$

or, in terms of $\left(t_{y}, t_{z}\right)$, coordinates of a point on the target projected onto a plane at right angles to the central incident ray (see Fig. 1)

$$
-0.06 \leqslant t_{z}-1.09 t_{y} \leqslant 0.06 \mathrm{~mm},
$$

where use has been made of $t^{\prime}=t_{z} / 2 \sin \left(\theta_{0}+\alpha\right)$ and $t=t_{y} / 2 \sin \left(\theta_{0}-\alpha\right)$.
The extreme values of $t$ and $t^{\prime}$ depend on the fraction of the $K \alpha_{1}$ line which one wants to reflect. Thus, if the spectral width to be reflected is the full width at half-maximum for the $\mathrm{K} \alpha_{1}$ line, which is $0.58 \mathrm{x} . \mathrm{u}$. for $\mathrm{Cu}$, then from Eqs. (1), (2), and (3),

$$
\begin{aligned}
& -0.10 \leqslant t \leqslant 0.14 \mathrm{~mm} \quad \text { i.e., }-0.13 \leqslant t_{y} \leqslant 0.17 \mathrm{~mm} \\
& -0.10 \leqslant t^{\prime} \leqslant 0.15 \mathrm{~mm} \quad \text { i.e., }-0.14 \leqslant t_{z} \leqslant 0.20 \mathrm{~mm} .
\end{aligned}
$$

From the inequalities (6) and (7), it is seen that the usable (projected) target area is a diagonal strip, approximately $0.09 \mathrm{~mm}$ wide and $0.45 \mathrm{~mm}$ long, inclined $47 \frac{1}{2}^{\circ}$ with the plane of the horizontal focal circle. For a $1-\mathrm{mm}^{2}$ target the usable strip is approximately $0.09 \mathrm{~mm} \times 1.4 \mathrm{~mm}$.

The general ray which emerges from point $S$ with coordinates $\left(t_{y}, t_{z}\right)$ will intersect the focal plane (plane through the ideal point focus and at right angles to the central ray) at a point with coordinates $\left(p_{y}, p_{z}\right)$ (see Fig. 1), the coordinates of the ideal point being $(0,0)$. The relationships between the two sets of coordinates are

$$
\begin{aligned}
& p_{y}=t_{z} \sin \left(\theta_{0}-\alpha\right) / \sin \left(\theta_{0}+\alpha\right) \\
& p_{z}=t_{y} \sin \left(\theta_{0}+\alpha\right) / \sin \left(\theta_{0}-\alpha\right),
\end{aligned}
$$

so that the inequalities to be imposed on $p_{y}$ and $p_{z}$ are almost the same as (6) and (7), namely,

$$
\begin{aligned}
& -0.06 \leqslant p_{z}-1.09 p_{y} \leqslant 0.06 \mathrm{~mm} \\
& -0.13 \leqslant p_{y} \leqslant 0.18 \mathrm{~mm} \\
& -0.14 \leqslant p_{z} \leqslant 0.18 \mathrm{~mm} .
\end{aligned}
$$

These inequalities indicate that the point focus is the image of the usable target area, namely, a strip $0.09 \mathrm{~mm}$ wide inclined at $47 \frac{1}{2}^{\circ}$ with the plane of the vertical focal circle. Furthermore, Eqs. (8) imply that it is possible to reduce the length of the point focus by stopping down the usable target area.

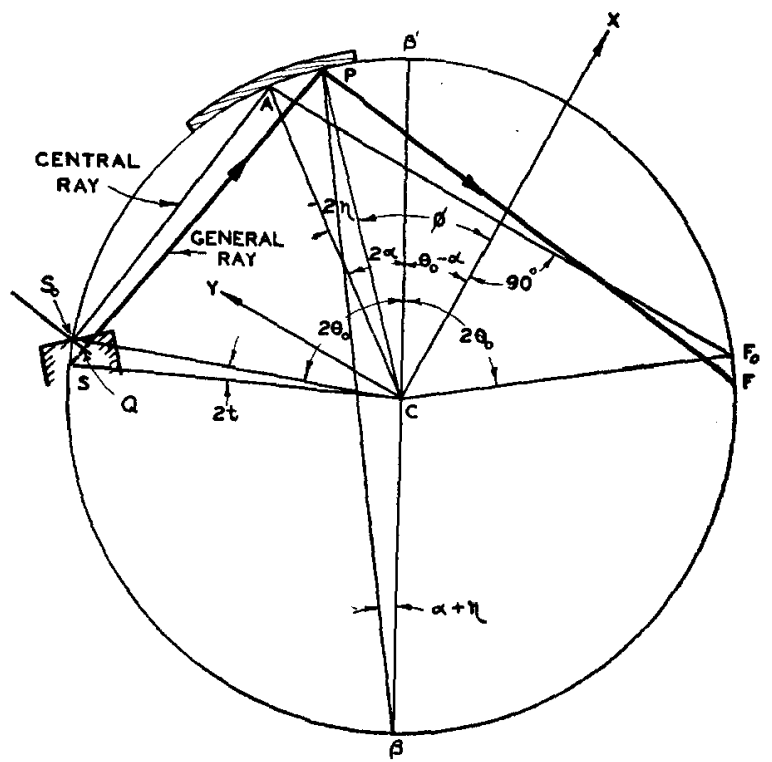

Fig. 4. Horizontal focal circle shown with its coordinate axes. 


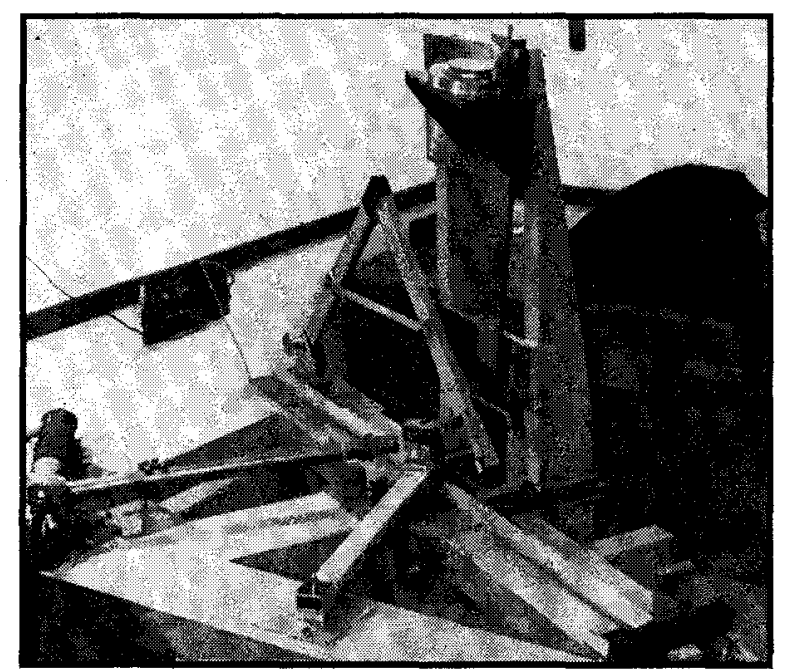

FIG. 5. The complete instrument with the exception of the sample holder and helium enclosure surrounding the two-crystal blocks.

An analysis, similar to the one above, carried out for the approximate focusing case, which is easier to realize in practice, shows that the point focus is aga in the image of the usable target area, namely, a diagonal strip, but in this case the dimensions of the strip are $0.19 \mathrm{~mm} \times 1.41 \mathrm{~mm}$ (for a $1-\mathrm{mm}^{2}$ target). Measurement of the exposed portion of a film placed in the focal plane of the monochromator for 2 minutes (the source of radiation being a Machlett tube operated at $35 \mathrm{pkv}$, $20 \mathrm{ma}$ ) shows it to be approximately a rectangular area $0.20 \times 1.40 \mathrm{~mm}$, a result which is consistent with the predicted value. This is at a distance of $75 \mathrm{~cm}$ from the second crystal or about $66 \mathrm{~cm}$ from the most distant position at which a scattering sample can be conveniently situated. Exposures of 20 or 30 hours, however, indicate that the size of the point focus is somewhat larger. This is probably due to the fact that the diffraction pattern of the quartz crystals extends over a wider angular range than was assumed in the above analysis.

\section{DESCRIPTION OF THE POINT FOCUSING MONOCHROMATOR AND ITS OPERATION}

The accompanying figure (Fig. 5) shows an over-all view of the instrument with the parts assembled for use, with the exception of the sample holder and the helium-filled housing surrounding the crystal clamping blocks and the $x$-ray paths. The $x$-ray tube is at the left and the two crystals may be seen, clamped between steel clamping blocks, at the center. A close-up view of the latter is shown in Fig. 6. The scattering sample is placed in the twice-reflected beam near the second crystal, and the film which records the diffraction pattern of the sample is held in place by a holder at the top of the vertical structure in such a way that the plane of the film is normal to the central ray and its center coincident with the point focus. With this arrangement, every point on a circle of specified radius (small in comparison to the sample-to-film distance) concentric with the central focal spot in the focal plane receives radiation which has been scattered under approximately the same angle. Thus aside from a negligible cosine correction arising from the fact that the rays in the direct beam are not parallel, the diffraction pattern represents truly the scattering property of the sample.

The cylindrical faces of the crystal clamping blocks (Fig. 6) are ground and lapped by a special method described by DuMond, Lind, and Cohen. ${ }^{9}$ This shop method has the advantages that it automatically insures (1) that the generators of the precision cylindrical clamping surfaces shall be truly normal to plane ground reference faces on the top and bottom of the crystal clamping blocks, and (2) that the radii of curvature on blocks for crystals $A$ and $B$ shall be closely equal.

Since there are twelve degrees of freedom involved in lining up the two crystals relative to the x-ray tube, it is necessary to have a systematic procedure whereby each degree of freedom is taken care of independently. To accomplish this, use is made of the reference surfaces mentioned above as well as the reference surface provided on the triangular table. The line focusing property of a single bent crystal permits the alignment of each crystal (independently of the other) on its supporting arm. This is done (first with the "vertical" crystal assembly and then with the "horizontal" one) in the horizontal position, thus allowing the $x$-ray tube to remain stationary. After each crystal is placed correctly on its supporting arm, the crystals (with their arms) are then placed as shown in Fig. 5, and the

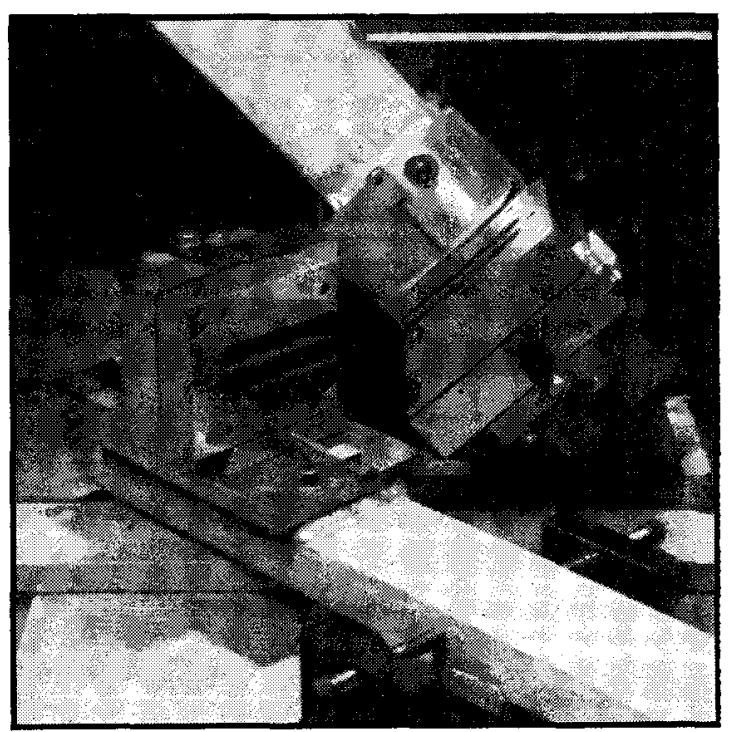

Fig. 6. Close-up view of the crystal blocks shown with the various adjusting screws.

${ }^{9}$ DuMond, Lind, and Cohen, Rev. Sci. Instr. 18, 617 (1947). 
instrument is corrected for astigmatism. This latter correction is made by pulling the second crystal toward away from the first along the line joining their centers.

The pertinent dimensions of the instrument are the following. The radius of curvature of the crystal laminas is approximately $120 \mathrm{~cm}$; hence the radius of each focal circle is $60 \mathrm{~cm}$. The maximum sample-to-film distance is about $66 \mathrm{~cm}$, and the cross section of the beam upon emergence from the second crystal is $2 \mathrm{~cm}$ $\times 2 \mathrm{~cm}$.

One of the present limitations of the instrument is the rather low intensity in the direct beam, which necessitates uncomfortably prolonged exposures for many scattering samples and becomes prohibitive in some cases. This difficulty arises from two effects. First, losses due to polarization occur because the two crystals behave in a manner similar to two Nicols which are crossed to almost complete extinction. (If the grazing angles $\theta$ and $\theta^{\prime}$ were $45^{\circ}$, complete extinction would occur.)

Second, not all the monochromatic radiation reflected by the first crystal strikes the second crystal at exactly the Bragg angle. Hence, only a portion of this radiation is reflected by the second crystal. This loss is inherent in the instrument and cannot be corrected easily.

There are at least two ways of improving the intensity: (1) by replacing the present $x$-ray tube (a commercial model) with one whose specific intensity is 10 to 20 times higher; (2) by replacing the quartz crystals with topaz. The latter crystals have better reflecting properties than quartz, and, furthermore, because the grazing angle (for (006) or (303) planes and $\mathrm{CuK} \alpha_{1}$ radiation) is smaller than for quartz, the losses resulting from polarization are not as large.

A compensating advantage of the instrument is the very favorable ratio of intensity in the primary pointfocused beam to the background intensity in the absence of a sample. This ratio is much better than in the case of a single-crystal monochromator. The diffuse background in a single-crystal monochromator comes chiefly from incoherent or diffuse scattering of the entire continuous spectrum incident on the crystal and also perhaps on parts of the crystal holder. This diffuse scattering is proportionately much more reduced by the second crystal reflection in the two-crystal point focusing monochromator than is the direct beam because the wavelength and direction of the rays are appropriate for the second Bragg reflection to a much greater extent in the once reflected beam than in the diffusely scattered beam.

Figure 7 is a photograph of the entire instrument with the working parts, including the entire $\mathrm{x}$-ray path from tube to diffraction camera, enclosed in a gas-tight helium-filled housing, Since the $\mathrm{x}$-ray path is $160 \mathrm{~cm}$

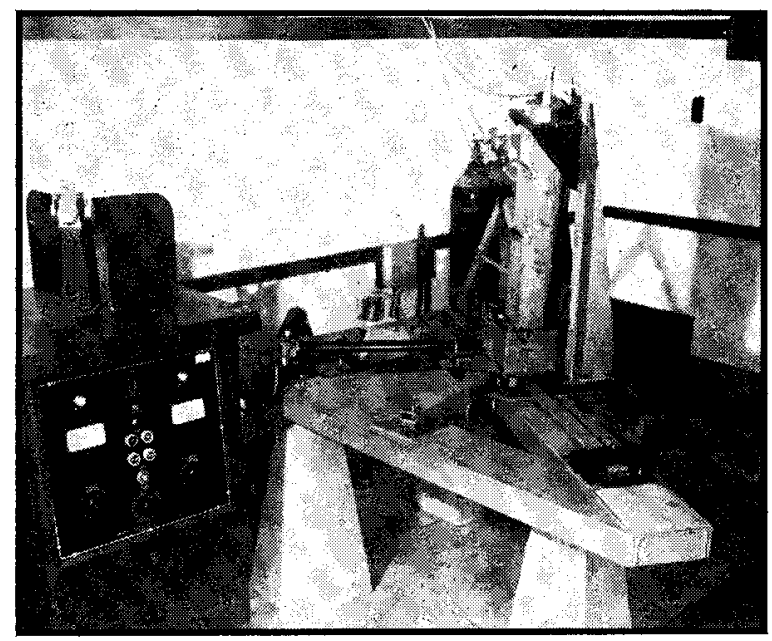

FIG. 7. The complete point focusing monochromator as used in low angle diffraction experiments.

long a considerable loss of $x$-ray intensity through air absorption is thus eliminated. The vertical part of this housing (slightly inclined toward the observer) which encloses that part of the beam which has undergone the second crystal reflection can be clearly seen. The scattering sample, in the form of a thin layer between Nylon films, is secured to a brass plate and can be introduced into the beam at any one of four clearly visible points, one of which, near the bottom, is quite close to the second crystal. The distances from sample to photographic film which these four locations permit are $66,36,16,6 \mathrm{~cm}$. These distances have been very carefully laid out in machining the slots for introduction of the brass plates (like drawers) into the beam. The selection of the best sample-to-film distance is made as a compromise between angular resolving power and exposure time required. Large diffraction patterns at fairly wide angles which will, in consequence, not have very great surface intensity because the scattered energy is distributed over a wide area are to be placed at the closer sample-to-film distances.

In conclusion we wish to express our gratitude to the ONR for their generous support and sponsorship of the development of this instrument and to Professor Linus Pauling of this Institute for his faith in and support of the project in recommending it to its present sponsors when its success was still problematical. We are also most grateful for the friendly advice and cooperation of Professor R. B. Corey of the C.I.T. Chemistry Department and to Professors J. Weigle and G. W. Beadle of the C.I.T. Biology Department for most helpful advice and discussions regarding possible problems suitable for the new instrument. 